\title{
Ocean jet instability: a model comparison
}

\author{
Jean-Michel Baey, Pascal Rivière and Xavier Carton
}

EPSHOM/CMO, Brest, 29275, France; baey@shom.fr

We investigate numerically the instability of three jets of the North Atlantic ocean (the GulfStream, the North Atlantic current and the Azores current), and the vortices resulting from this instability. For this problem, we compare two hydrodynamical models (the shallow-water and the quasi-geostrophic equations) which we implement in identical conditions (two-layer stratification). First, both models are linearized and growth rates are calculated for zonal, normal-mode perturbations on such jets. When we vary the Burger and the Rossby numbers of the jets and the wavenumber of the perturbations, the two linear models yield fairly similar results. Secondly, both nonlinear models (discretized on a fixed regular grid) are used to compute the finite-amplitude evolutions of the perturbed jets. These evolutions are qualitatively similar in both models, but the dynamical features (jet and vortices) are longer-lived in the shallow-water than in the quasigeostrophic model. Also, only the former model exhibits an asymmetry between cyclones and anticyclones, which is physically explained.

\section{Introduction}

The large-scale, wind-driven currents of the North Atlantic ocean, between $20^{\circ} \mathrm{N}$ and $60^{\circ} \mathrm{N}$, can be idealized on part of their courses as thin, zonal ${ }^{1}$ jets. Among them are the Gulf-Stream (GS) in the western basin, the North Atlantic current (NAC) and the Azores current (AC) in the eastern basin. Though the GS, NAC and AC have different characteristics (see table 1), their velocity profiles are sheared horizontally and vertically, and they are unstable. Thus they form meanders and vortices, which detach from the jets and drift away (Kontoyannis \& Watts, 1994; Lee, 1994).

Such ocean jets are flows with small (vertical to horizontal) aspect ratios, in an incompressible fluid. An appropriate model for their study is the "shallow-water" (SW) equations. Up to now, jet instability has been studied mostly with a "quasi-geostrophic" (QG) equations (Flierl et al., 1987, 1998), a subset of the SW equations valid for small Rossby numbers and order-one Burger numbers. The present paper studies ocean jet instability in both QG and SW models, for a variety of Rossby and Burger numbers. Application to the GS, NAC and AC is performed. Vortex formation from unstable jets is more specifically analyzed.

The paper is organized as follows: in section 2, the QG and SW models are presented and linearized. In section 3 , growth rates are computed for normal mode perturbations on the jets; they are compared between the two models. In section 4, nonlinear QG and SW models are used to determine numerically the finite-amplitude evolutions of the perturbed jets (GS, NAC and AC). Asymmetry in cyclone versus anticyclone formation in the shallow-water model and conclusions are presented in sections 5 and 6 .

\footnotetext{
${ }^{1}$ Zonal currents flow along parallels
} 


\section{Model equations and linear stability theory}

\subsection{Model equations}

The GS, NAC and AC are essentially horizontal flows, with a small aspect ratio, in an incompressible, hydrostatic, Boussinesq, two-layer fluid. Jet dynamics are thus governed by the shallow-water equations, which are written (in the absence of forcing and of dissipation):

$$
\begin{gathered}
\partial_{t} u_{j}+u_{j} \partial_{x} u_{j}+v_{j} \partial_{y} u_{j}-f v_{j}=\frac{-1}{\rho_{0}} \partial_{x} p_{j} \\
\partial_{t} v_{j}+u_{j} \partial_{x} v_{j}+v_{j} \partial_{y} v_{j}+f u_{j}=\frac{-1}{\rho_{0}} \partial_{y} p_{j} \\
\partial_{t} h_{j}+\partial_{x}\left(h_{j} u_{j}\right)+\partial_{y}\left(h_{j} v_{j}\right)=0
\end{gathered}
$$

Here the $x$ - and $y$ - directions are the longitudinal and latitudinal axes; $j=1,2$ is the (upper,lower) layer index; $h_{j}=H_{j}+(-1)^{j} \eta$ is the local thickness of layer $j ; \eta$ is the upward displacement of the density interface between the two layers; $H=H_{1}+H_{2}$ is the ocean depth; $\rho_{0}$ is a vertically-averaged density, and we retain the $\beta$-effect in the Coriolis term ${ }^{2}, f=f_{0}+\beta y$. The hydrostatic balance is $p_{2}-p_{1}=\left(\rho_{2}-\rho_{1}\right) g \eta$. The reduced gravity is $g^{\prime}=g\left(\rho_{2}-\rho_{1}\right) / \rho_{0}$. By taking the curl of eq.1-2 and replacing the velocity divergence in eq.3, a conservation equation is formed for the potential vorticity $Q_{j}$ :

$$
\left(\partial_{t} v_{j}+u_{j} \partial_{x}+v_{j} \partial_{y}\right) Q_{j}=0, \quad Q_{j}=\frac{\partial_{x} v_{j}-\partial_{y} u_{j}+f}{h_{j}}
$$

All these equations can be scaled, using length, depth and velocity scales $L, H$ and $U$, by the Rossby number $R_{o}=U / f_{0} L$, the Burger number $B u=g^{\prime} H_{1} H_{2} /\left(f_{0}^{2} L^{2} H\right)$ and the dimensionless $\beta$-effect $\bar{\beta}=\beta L / f_{0}$. The Burger number is also related to the deformation radius $R_{d}$ by $B u=R_{d}^{2} / L^{2}$. In dimensionless terms, the potential vorticity is:

$$
Q_{j}=\frac{f_{0}}{H_{j}} \frac{\left(1+\bar{\beta} y+R o \zeta_{j}\right)}{(1+R o \eta / B u)}
$$

with the relative vorticity $\zeta_{j}=\partial_{x} v_{j}-\partial_{y} u_{j}$.

For weak Rossby numbers and order-one Burger numbers, geostrophic balance (between the horizontal pressure gradient and the Coriolis acceleration) is attained so that the horizontal velocity is nondivergent at first order in $R o: u_{j}=-\partial_{y} \psi_{j}, v_{j}=\partial_{x} \psi_{j}$. The planetary vorticity gradient is assumed weak and scaled as $\bar{\beta}=R o \beta^{*}$. The conservation of potential vorticity is now:

$$
\begin{gathered}
\frac{d\left(q_{j}+\beta^{*} y\right)}{d t}=\left[\partial_{t}+J\left(\psi_{j}, \cdot\right)\right] q_{j}+\beta^{*} \partial_{x} \psi_{j}=0, \\
q_{j}=\frac{H_{j} Q_{j}}{f_{0}}-1=\nabla^{2} \psi_{j}+\frac{H_{j}}{B u H}\left(\psi_{k}-\psi_{j}\right)
\end{gathered}
$$

with $k=3-j$. We have used the hydrostatic balance to relate $\eta$ and $\psi_{j}$.

\footnotetext{
${ }^{2} f=2 \Omega \sin (\theta)$, where $\Omega$ is the rotation rate of the Earth, $\theta$ is the latitude, $y=R_{e} d \theta$ and $R_{e}$ is the radius of the Earth
} 


\section{$2.2 \quad$ Linear stability theory}

The basic jet is zonal (here eastward) and in geostrophic balance. Its velocity profile is:

$$
\bar{u}_{j}(y)=\frac{U_{j}}{\cosh ^{2} \frac{y}{L}}, \quad U_{2}=\alpha U_{1}
$$

Here we define more specifically $R o=U_{1} / f_{0} L$ and the Froude number $F r=R o / \sqrt{B u}$.

In both models, the total flow is the superposition of the jet and of a normal-mode perturbation:

$$
\Gamma_{j}=\bar{\Gamma}_{j}(y)+\epsilon \cdot \mid \operatorname{Re}\left[\Gamma_{j}^{\prime}(y) \cdot e^{i k(x-c t)}\right]
$$

with $\Gamma_{j}=\left(u_{j}, v_{j}, h_{j}, p_{j}\right)$, in SW, and $\left(q_{j}, \psi_{j}\right)$ in QG dynamics; note that $\bar{v}_{j}=0 ; k$ is the wavenumber, $c=c_{r}+i c_{i}, \sigma=k c ; \operatorname{Im}(\sigma)$ is the growth rate.

For the shallow-water model, equations $(1,2,3)$ are linearized to finally yield:

$$
\begin{gathered}
\frac{R o\left(\sigma+k \bar{u}_{j}\right) \bar{h}_{j}}{D_{j}} \frac{d^{2} p_{j}^{\prime}}{d y^{2}}-\frac{R o\left(\sigma+k \bar{u}_{j}\right)}{D_{j}}\left[\frac{D_{j}^{\prime}}{D_{j}} \bar{h}_{j}-\frac{d \bar{h}_{j}}{d y}\right] \frac{d p_{j}^{\prime}}{d y} \\
-\frac{k}{D_{j}}\left[\left(\bar{\beta}+k R o\left(\sigma+k \bar{u}_{j}\right)\right)-\frac{D_{j}^{\prime}}{D_{j}}(1+\bar{\beta} y) \bar{h}_{j}+(1+\bar{\beta} y) \frac{d \bar{h}_{j}}{d y}\right] p_{j}^{\prime} \\
=(-1)^{j}\left(\sigma+k \bar{u}_{j}\right) \frac{F r^{2}}{R o}\left(p_{1}^{\prime}-p_{2}^{\prime}\right)
\end{gathered}
$$

with

$$
\begin{gathered}
D_{j}=R^{2}\left(\sigma+k \bar{u}_{j}\right)^{2}+R o(1+\bar{\beta} y) \frac{d \bar{u}_{j}}{d y}-(1+\bar{\beta} y)^{2} \\
D_{j}^{\prime}=\operatorname{Ro}(1+\bar{\beta} y) \frac{d^{2} \bar{u}_{j}}{d y^{2}}+\operatorname{Ro}\left[\bar{\beta}+2 \operatorname{Rok}\left(\sigma+k \bar{u}_{j}\right)\right] \frac{d \bar{u}_{j}}{d y}-2 \bar{\beta}(1+\bar{\beta} y)
\end{gathered}
$$

The domain is a wide zonal channel and the boundary conditions are no normal flow at the northern and southern walls:

$$
R o\left(\sigma+k \bar{u}_{j}\right) \frac{d p_{j}^{\prime}}{d y}-k(1+\bar{\beta} y) p_{j}^{\prime}=0
$$

The growth rates are insensitive to the width of the channel, provided it is much larger than the jet width. This problem is discretized in $y$ and the growth rates of the perturbations are computed by a Newton-Raphson method.

For the quasi-geostrophic model, the linearized potential vorticity equation is:

$$
\left(\bar{u}_{j}-c\right) q_{j}^{\prime}+\left[\frac{d \bar{q}_{j}}{d y}+\beta^{*}\right] \psi_{j}^{\prime}=0
$$

The boundary conditions are $\psi_{j}^{\prime}=0$ at the northern and southern walls. Once discretized in $y$, this equation forms a generalized eigenvalue problem solved by a matrix method. 

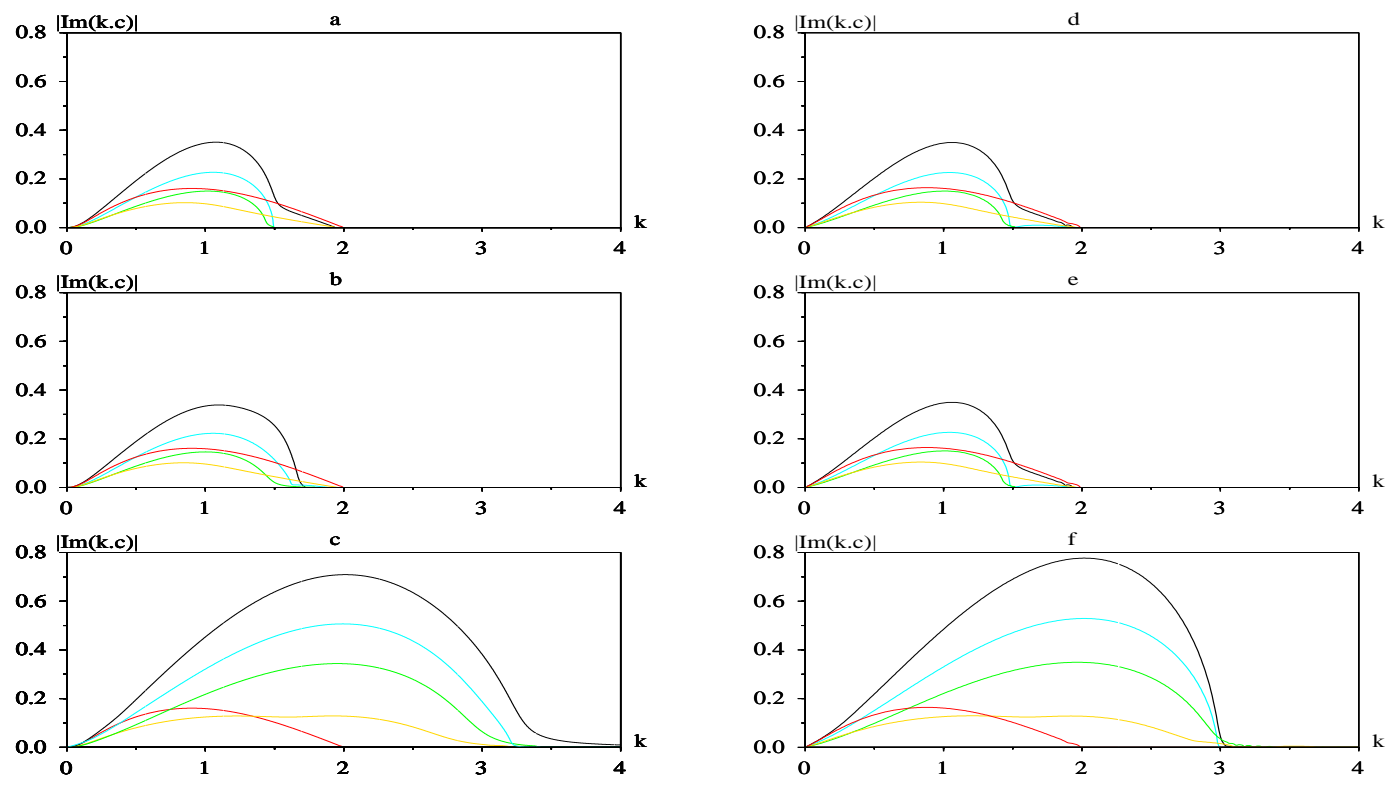

Figure 1: Growth rate of zonal normal-mode perturbations versus wavenumber in the SW model (a,b,c) and in the QG model (d,e,f); $R o=0.06, B u=0.36(\mathrm{a}, \mathrm{d}) ; R o=0.3, B u=0.36(\mathrm{~b}, \mathrm{e})$; $R o=0.06, B u=0.09(\mathrm{c}, \mathrm{f})$. The following colors are used for $\alpha=U_{2} / U_{1}$ : red $(\alpha=1.0)$, yellow ( $\alpha=0.4)$, green $(\alpha=0.0)$, blue $(\alpha=-0.4)$, black $(\alpha=-1.0)$.
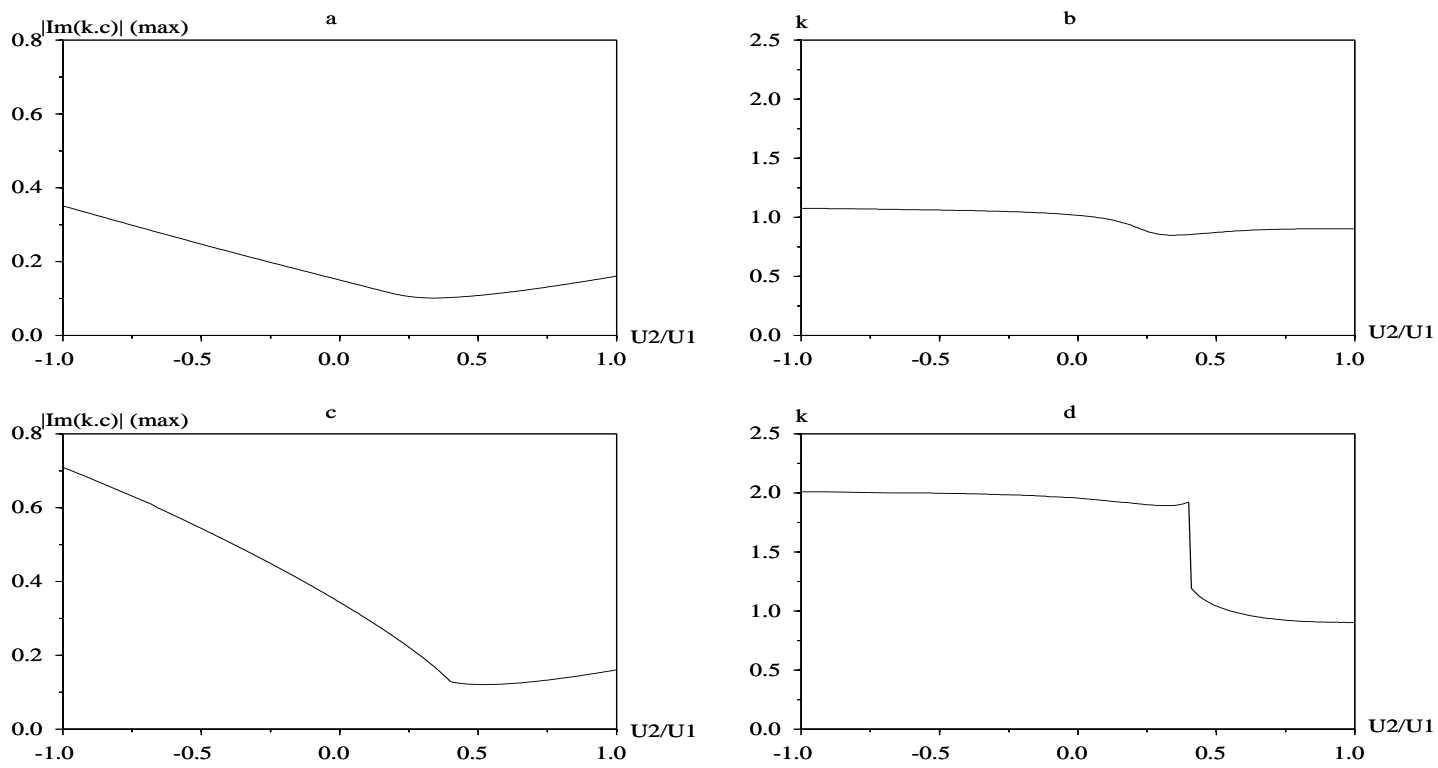

Figure 2: Maximum growth rates versus $\alpha$ for $B u=0.36$ (a) and for $B u=0.09$ (c); the corresponding wavenumbers are shown in (b) and (d). These results have been obtained with the SW model, for $R o=0.06, \bar{\beta}=0.0$. 

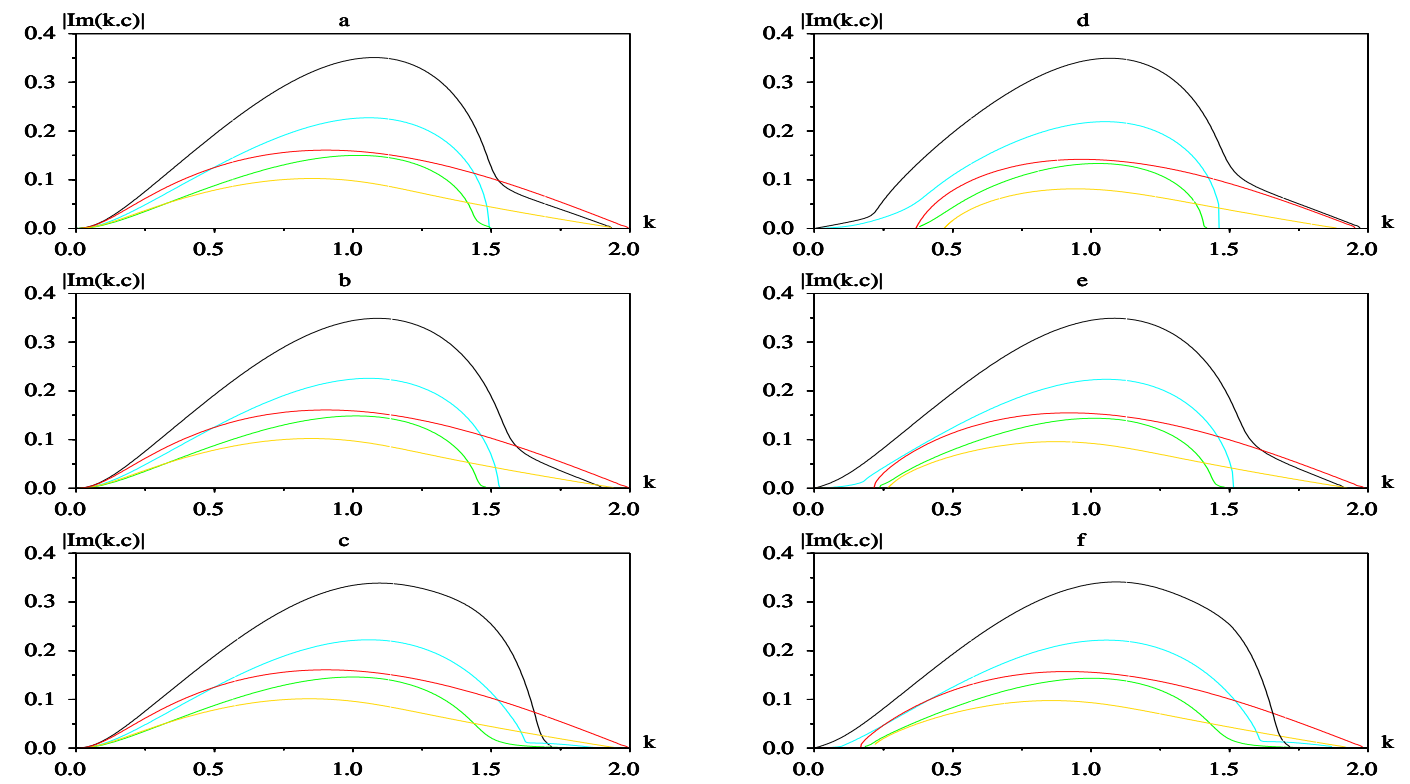

Figure 3: Growth rates of normal-mode perturbations versus wavenumber in the SW model for $\bar{\beta}=0.0$, and $R o=0.06$ (a), $R o=0.18$ (b), $R o=0.3$ (c); plots (d,e,f) correspond to (a,b,c) for $\bar{\beta}=0.0052$. Here $B u=0.36$. The color code for $\alpha$ is identical to that of fig. 1 .
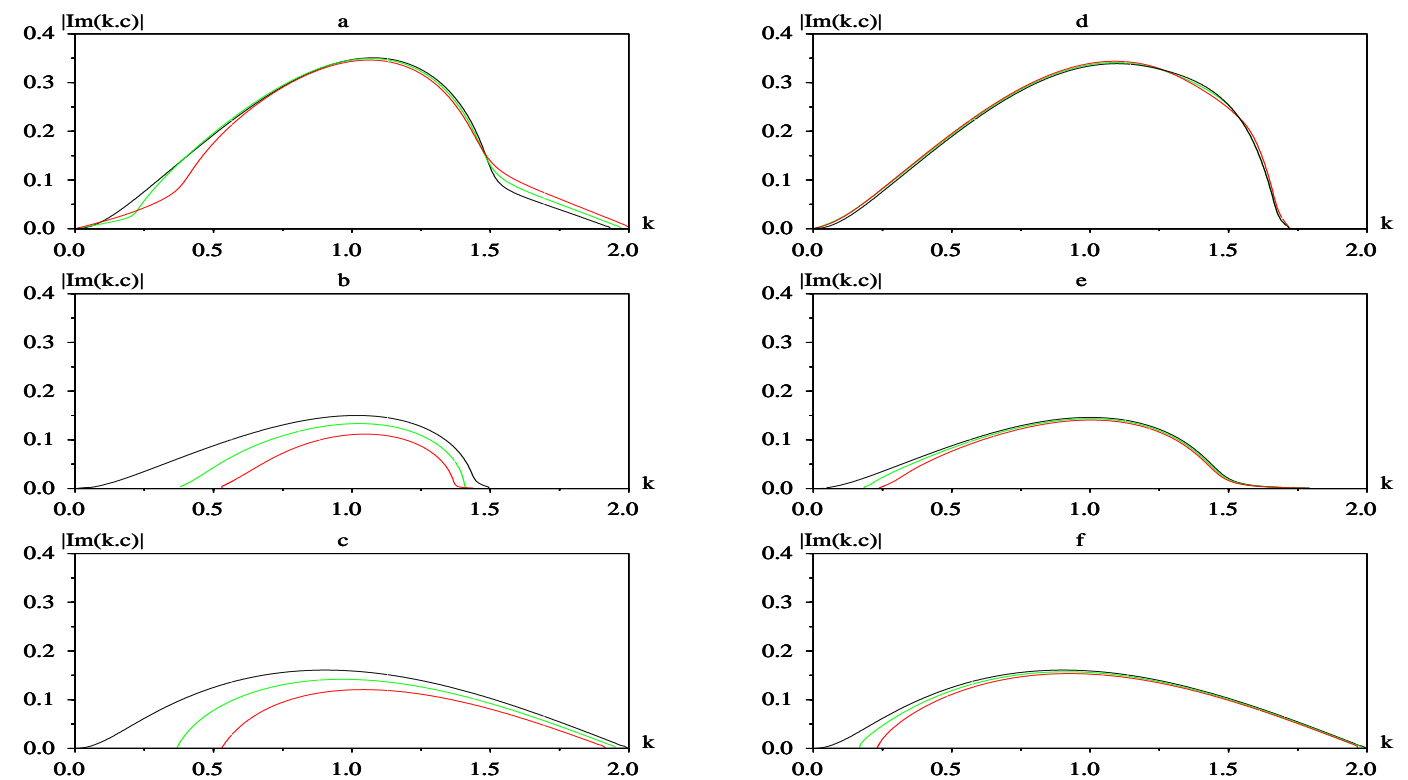

Figure 4: Growth rates of normal-mode perturbations versus wavenumber in the SW model for $R o=0.06$ and $\alpha=-1.0$ (a), $\alpha=0.0$ (b), $\alpha=1.0$ (c); plots (d,e,f) correspond to (a,b,c) for $R o=0.3$. Here $B u=0.36$. The red curve is $\bar{\beta}=0.0104$, the green curve is $\bar{\beta}=0.0052$, the black curve is $\bar{\beta}=0.0$. 


\section{Growth rates for normal-mode perturbations}

Table 1 shows that the oceanic jet characteristics are quite different. For their linear stability analysis, we impose common physical parameters: $H_{1} / H=0.2, f_{0}=10^{-4} \mathrm{~s}^{-1}, L=50 \mathrm{~km}$. The maximum velocity in the upper layer varies from 0.3 to $1.5 \mathrm{~m} . \mathrm{s}^{-1}$, leading to $R o \in[0.06,0.3]$. The jet baroclinicity is chosen as $\alpha=U_{2} / U_{1} \in[-1,+1]$, and two deformation radii are considered, $R_{d}=15 \mathrm{~km}$ and $R_{d}=30 \mathrm{~km}$. Thus the two values of the Burger number considered here are $B u=0.09$ and $B u=0.36$. Finally, wavelengths are defined by $\lambda=2 \pi L / k=315 \mathrm{~km} / k$, where $k$ is the wavenumber.

Figure 1 compares the growth rates obtained with the QG and SW models. A striking similarity between them is observed whatever $\alpha, B u, R o$. Slight differences are noticed only for large values of $(1-\alpha) R o / B u$ (which quantifies the frontal aspect of the density interface). This quantity reaches 0.9 on the black and blue curves of Fig. 1b,c,e,f. Indeed, the QG model is not appropriate to describe frontal flows (when $R o \sim B u$ ).

When $B u=0.36$, one family of unstable eigenmodes is dominant whatever $\bar{\beta}$ and $R o$, except for very baroclinic jets $(\alpha<-0.4)$. Fig. 2a,c shows that the instability is minimum for $\alpha \sim 0.3$, that is, close to surface-intensified jets. For $\alpha<0.3$, the most unstable wavenumber is $k=1$ (Fig. $2 \mathrm{~b}$ ); this corresponds to $315 \mathrm{~km}$-wavelengths, comparable with Gulf-Stream meander observations (Lee, 1994). More barotropic jets $(\alpha>0.3)$ are most unstable on slightly longer wavelengths.

Fig 3a,b,c shows that for $\bar{\beta}=0$, the growth rates are proportional to $R o$ (they are normalized by $U_{1} / L$ on the plot). This is less true for $\bar{\beta}=0.0052$, the normalized value of $\beta$ on Earth (see Fig. 3d,e,f).

Fig. 4 shows that large values of $\bar{\beta}$ have a stabilizing effect, especially on long waves (see also Flierl et al., 1998). This stabilizing influence is maximum for small Ro and when $\alpha \geq 0$ (Fig. 4b,c). Note also that for weak baroclinic jets $(R o=0.06, \alpha=-1.0)$, growth rates increase slightly with $\bar{\beta}$ for $k>1.5$. This corresponds to the drift of the most unstable modes towards smaller wavelengths, when $\bar{\beta}$ increases.

When $B u=0.09$, growth rates present a few differences with the previous case:

- they are non-zero for $k \in[0 ; 3]$ or $\lambda>105 \mathrm{~km}$, except when the jet is barotropic (Fig. 1c,f);

- baroclinic jets are more unstable than for $B u=0.36$, especially on short waves (Fig. 1c,f and Fig. 2c);

- the evolution between barotropic and baroclinic jet instability is discontinuous (Fig. 2d).

The three jets of the north Atlantic ocean are surface-intensified $(\alpha \sim 0)$. From the computed growth rates, the NAC $(B u=0.09, R o=0.06)$ is unstable on shorter wavelengths than the GS $(B u=0.36, R o=0.3)$ and that the $\mathrm{AC}(B u=0.36, R o=0.06)$. The GS is more unstable than the NAC, and much more unstable than the AC.

\section{Nonlinear evolutions of the three jets}

The nonlinear simulations are performed with numerical gridded models of (a) the shallow-water equations $(1,2,3)$, and (b) the QG equation (4). The SW model geometry is a zonally-periodic channel, with length and width $1130 \mathrm{~km}$. The grid size is $4 \mathrm{~km}$. On the northern and southern solid walls, free-slip boundary conditions are applied. The QG model is biperiodic with identical 


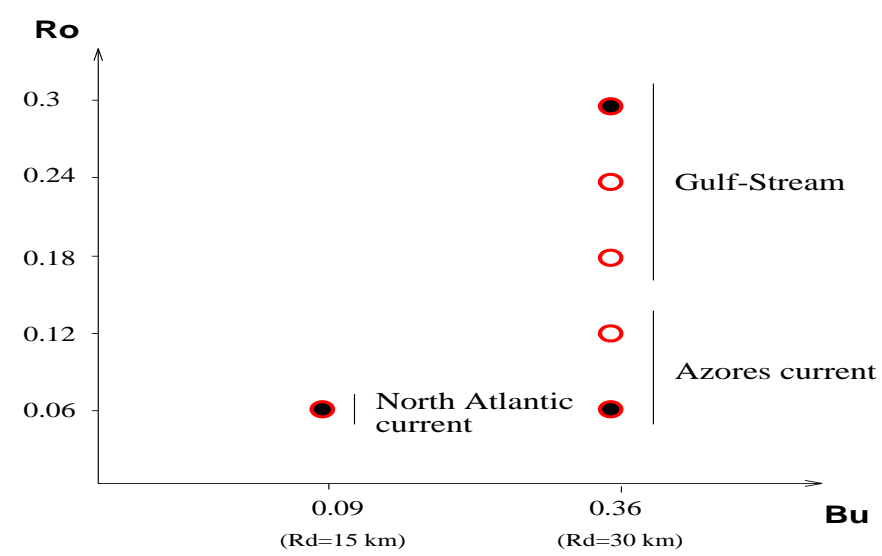

Figure 5: Nonlinear simulations performed with both models, in the $(B u, R o)$ plane; the corresponding internal radius of deformation $\left(R_{d}\right)$ is given. The three simulations detailed in section 4 are indicated by a black dot.

parameters. It has been checked that the physical results are independent of these numerical choices. Both models use the same physical parameters. Biharmonic viscosity removes small scale vorticity arising from the enstrophy cascade; the dissipative time scale is 100 days. In every nonlinear simulation, we set $H_{1}=800 \mathrm{~m}, H_{2}=3200 \mathrm{~m}, f_{0}=10^{-4} \mathrm{~s}^{-1}, \beta=1.7310^{-11} \mathrm{~m}^{-1} \mathrm{~s}^{-1}$, $L=50 \mathrm{~km}$, and $\alpha=0$. The Burger $B u$ and Rossby Ro numbers are varied.

In the $(R o, B u)$ plane, six cases are studied nonlinearly (Fig. 5), with characteristics close to: the Gulf-Stream (large Ro and large $B u$ ), the Azores current (low Ro and large $B u$ ) and the North Atlantic current (low $R o$ and low $B u$ ). Both models are initialized with the zonal jet defined in section 2.2 to which is added a weak random-noise perturbation.

\subsection{The Gulf-Stream}

In the GS, upper-layer currents vary between $U_{1}=0.9$ and $1.5 \mathrm{~m} . \mathrm{s}^{-1}$. Here we have set $U_{1}=$ $1.5 \mathrm{~m} . \mathrm{s}^{-1}(R o=0.3)$ and a deformation radius $R_{d} \sim 30 \mathrm{~km}(B u=0.36)$. Fig. 6 shows time-series of the interface elevation between layers in numerical simulations of the nonlinear QG and SW models. During the first stage of the evolution (including the linear stage), both models show the growth of regular meanders, with a wavelength close to $280 \mathrm{~km}$ (fig. 6.a.b). This wavelength was determined as the most unstable one (with the same growth rate) by the linear stability analysis. However, after this first stage and until the end of the simulation, the nonlinear evolutions of the jet differ in the two models (fig. 6.c.d) : vortex formation is clearly symmetric (in size and number) in the QG model, whereas we observe a pronounced asymmetry in the SW model with fewer, but larger anticyclones north of the jet and smaller and more numerous cyclones south of the jet. Moreover, the final jet exhibits large meanders in the SW model (with $\lambda \sim 1130 \mathrm{~km}$ ), while smaller meanders are observed in the QG model $(\lambda \sim 380 \mathrm{~km})$. This denotes a stronger nonlinear energy cascade towards large scales in the SW model than in the QG model. 


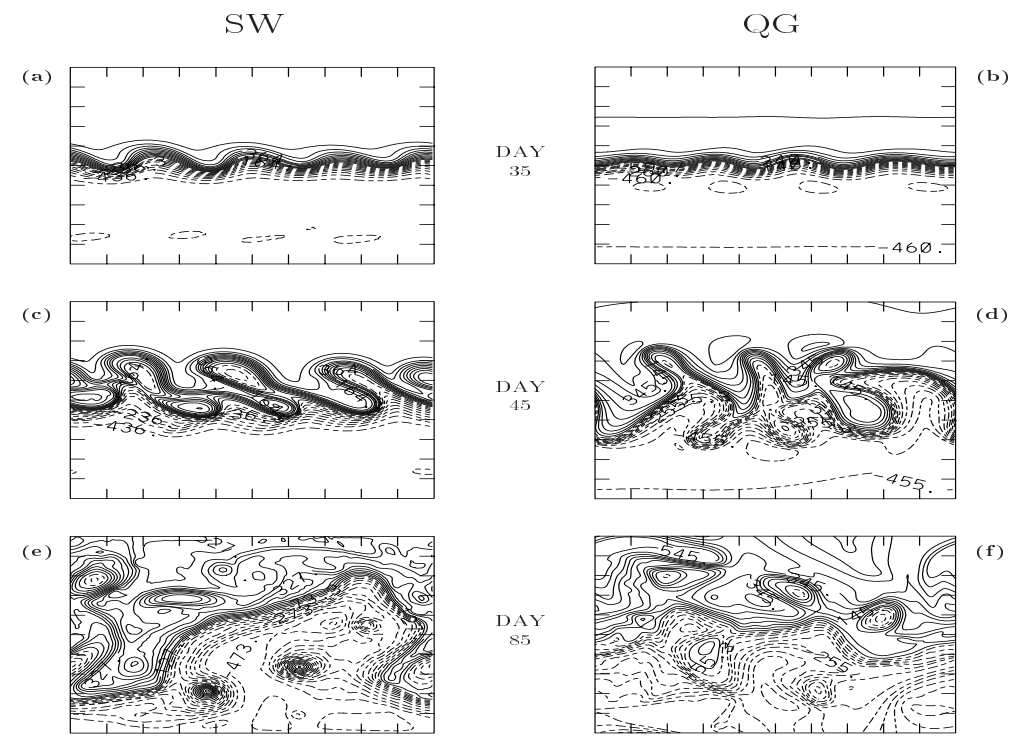

Figure 6: Nonlinear simulations corresponding to the Gulf-Stream $(R o=0.3, B u=0.36)$; snapshots of the interface elevation at days 35, 45 and 85. SW model (a,c,e) and QG model (b,d,f). Contour intervals in meters.

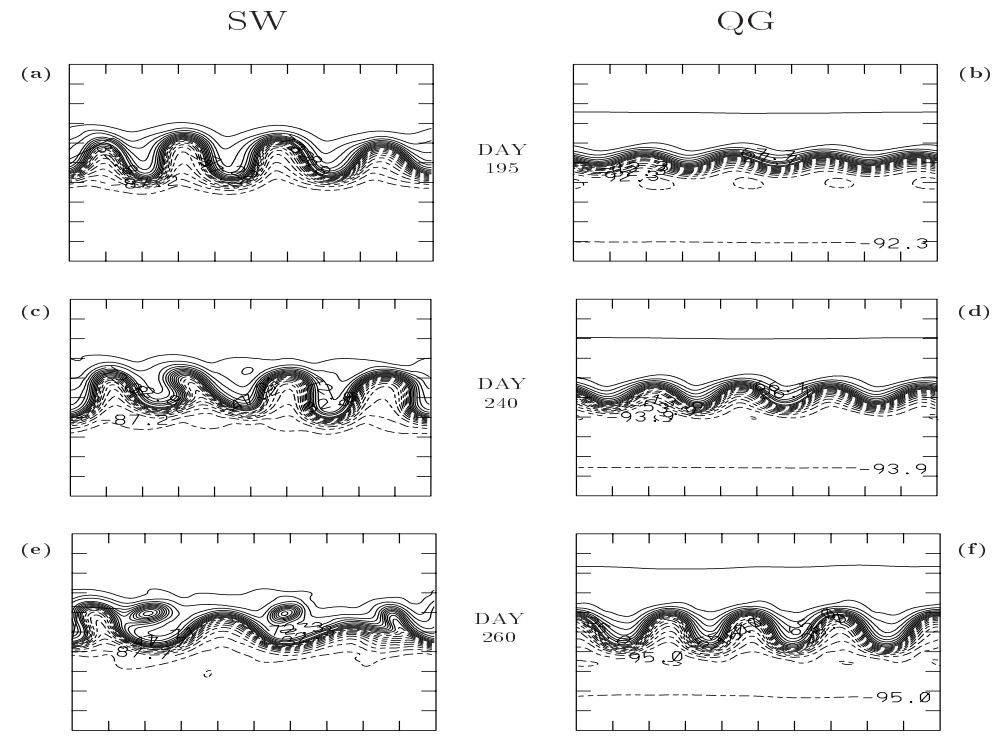

Figure 7: Nonlinear simulations corresponding to the Azores current $(R o=0.06, B u=0.36)$; snap-shots of the interface elevation at days 195, 240 and 260. SW model (a, c,e) and QG model $(\mathrm{b}, \mathrm{d}, \mathrm{f})$. Contour intervals in meters. 


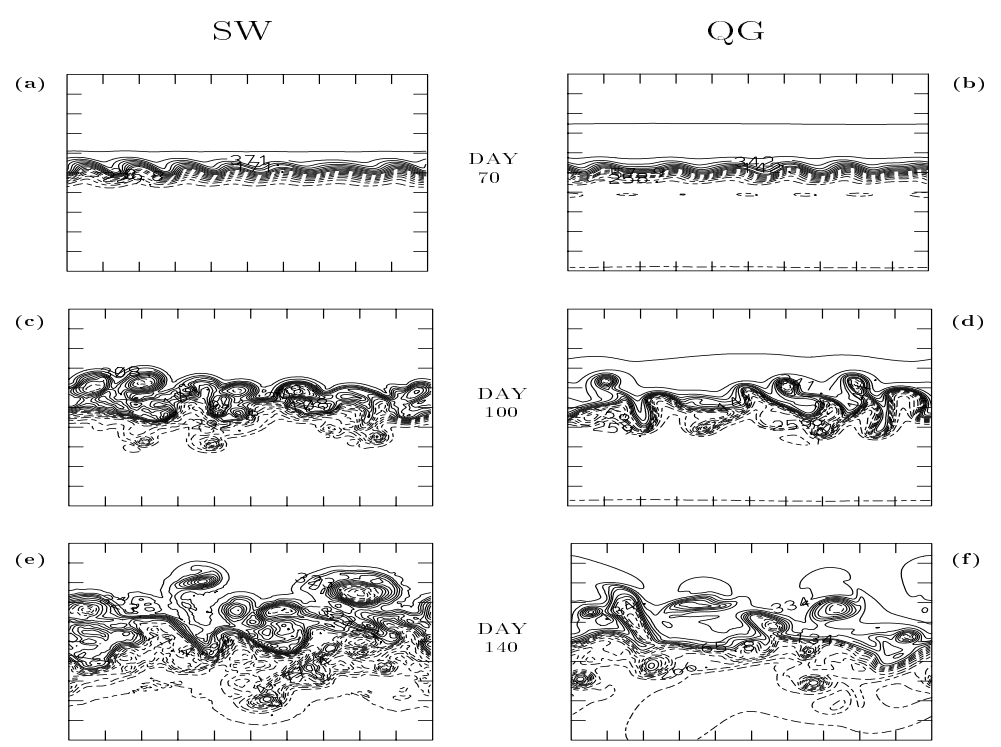

Figure 8: Nonlinear simulations corresponding to the North Atlantic current $(R o=0.06, B u=$ 0.09); snap-shots of the interface elevation at days 70, 100 and 140. SW model (a,c,e) and QG model $(\mathrm{b}, \mathrm{d}, \mathrm{f})$. Contour intervals in meters.

\subsection{The Azores current}

For the $\mathrm{AC}$, the deformation radius is again $R_{d} \sim 30 \mathrm{~km}(B u=0.36)$, but typical velocities are only $U_{1}=0.3-0.6 \mathrm{~m} . \mathrm{s}^{-1}(R o=0.06,0.12)$. Here we set $U_{1}=0.3 \mathrm{~m} . \mathrm{s}^{-1}(R o=0.06)$. Fig. 7 shows three snapshots of the interface elevation in both SW and QG models. Obviously, the AC is less unstable than the GS; thus, the first stage of the instability shows the emergence of less energetic meanders, which still have the same wavelength as those of the Gulf-Stream (Fig. 7a,b). Wavelengths and growth rates are in agreement with the linear stability analysis.

When nonlinear effects develop (Fig. 7c,d), we observe an asymmetry in vortex polarity in the SW model: only large anticyclones are produced north of the jet. On the contrary, in the QG model, no vortices are produced and the jet remains symmetric.

A normal-mode decomposition of the perturbation on the jet is then performed at regular timeintervals. After similar linear stages, where mode $4(\lambda=280 \mathrm{~km})$ grows the fastest, the nonlinear stages are different in the two models, since mode $3(\lambda \sim 380 \mathrm{~km})$ finally dominates in the SW model, evidencing stronger nonlinear energy exchanges in this model (not shown here).

\subsection{The North Atlantic current}

This jet is characterized by $U_{1}=0.3 \mathrm{~ms}^{-1}(R o=0.06)$ and $R_{d}=15 \mathrm{~km}(B u=0.09)$. Fig. 8 shows three snap shots of the interface elevation in both SW and QG models. Because of the lower stratification, the NAC is more unstable than the AC, and on shorter wavelengths, for the same value of $R o$. Indeed meanders are more energetic here and have a wavelength close to $160 \mathrm{~km}$, 
Jean-Michel Baey, Pascal Rivière and Xavier Carton

in agreement with the linear stability analysis (Fig. 8a,b). Also, these figures show no difference between the two models in the linear stage, again as predicted by the linear stability analysis.

Differences between the two models arise when nonlinearities become important (Fig. 8c,d). Many vortices are produced by the jet (Fig. 8e,f). As for the GS, anticyclones are larger than cyclones in the SW model, but here anticyclones are more numerous. In the QG model, no significant asymmetry between vortices is observed.

\section{The effects of nonlinearities and cyclone-anticyclone asymme- try}

Two striking features of these numerical simulations are:

- the robustness and nonlinearity of jets and vortices in the SW model, compared with the QG model;

- the north-south asymmetry of meanders and vortices in the SW model, contrary to the QG model.

These results contrast with the linear stability analysis, which did not reveal any significant difference between the two dynamics. Thus, they originate in nonlinear effects.

The first point is clearly explained by the validity of the SW equations in regimes of large $R o$ and $R o / B u$, which describe intense, frontal flows. Indeed the $\mathrm{AC}(R o=0.06, B u=0.36)$ forms vortices in the SW dynamics, while it does not in the QG model. Also, the number of vortices produced increases with $R o$ in the SW model, but not in the QG one.

For the second point, we note that the QG model does not exhibit any statistical difference between cyclones and anticyclones, neither in size, nor in number. On the contrary, in the SW model, anticyclones are larger than cyclones. This difference can be explained by the meridional variation of the stratification: north of the jet, the upper layer is thinner and the lower one thicker than south of the jet. Therefore, the northern deformation radius is smaller. Since vortices tend to adopt the size of the deformation radius, anticyclones, which contain water from south of the jet with a larger deformation radius, are larger than cyclones which contain northern water (see also Alves, 1996).

Numerical experiments also show that anticyclones are more numerous than cyclones for the AC and NAC, and slightly less numerous for the GS. This difference in number, if confirmed by further numerical experiments, can result from the nature of the instability. It is essentially baroclinic for the AC and NAC, and mixed barotropic-baroclinic for the GS (see also Flierl et al., this volume). We also recall that baroclinic instability is favored by small deformation radii, found north of the jets (and conversely for the barotropic instability). Therefore, baroclinic instability can be favored north of the $\mathrm{AC}$ and $\mathrm{NAC}$, forming more anticyclones, and barotropic instability can prevail south of the GS, leading to more cyclones.

\section{Conclusions}

The aim of this paper was to study zonal jet instability, with application to the North Atlantic ocean, and to compare two dynamical models in that respect. The QG and SW models proved similar for the linear stability analysis of the jets, but quite different for their nonlinear evolu- 
tions. First, jets and vortices are more robust and longer-lived in nonlinear SW simulations, with a particular consequence for the AC, which produces vortices only in the SW model. Secondly, in this model, a cyclone-anticyclone asymmetry appears, leading to larger anticyclones, and more numerous anticyclones for the NAC and AC. This asymmetry can be explained as a consequence of the different stratification north and south of the jet modifying the deformation radii and the intensity of baroclinic and barotropic instabilities.

However, this asymmetry must be confirmed, since recent numerical simulations have shown its sensitivity to $\beta^{*}$ and $L$ values. In particular, for $L=30 \mathrm{~km}$ (a lower limit from a realistic point of view), a reverse asymmetry was observed in the Gulf-Stream and North Atlantic current simulations (see also Bush et al., 1994). This sensitivity of cyclone-anticyclone asymmetry to $L$ could be explained by the presence of critical layers, which can modify the nonlinear stability of the jet, and which depend on the jet width (Lee and Held 1991). To quantify the competition between baroclinic and barotropic instabilities and to investigate the effects of physical parameters on the asymmetry, energy transfers between the jet and the perturbation must be calculated.

Observations tend to show that this asymmetry in the number of anticyclones is more marked for the NAC which is a frontal jet. In the ocean indeed, other effects, not taken into account here, can modify this asymmetry. Among them, and central, is bottom topography. For instance, the Blake plateau north of the GS is known to accelerate the decay of the anticyclones. Further work will incorporate these effects.

\section{Ackowledgements}

Funding for this study was provided by DGA and SHOM (France) under contracts FRE-91009401 and PEA-982401.

\section{References}

1 Alves, M.: Instability dynamics of a subtropical jet : the Azores Front-Current system case (FCA). PhD Thesis, Laboratoire de Physique des Océans, Université de Bretagne Occidentale, Brest, France (1996), $229 \mathrm{pp}$.

2 Bush A.B., McWilliams J.C. And W.R. Peltier: The formation of oceanic eddies in symmetric and asymmetric jets. Part I: Early-time evolution and bulk eddy transports. J. Phys. Oceanogr. 25 (1994), 1959-1979.

3 Emery W.J., Lee W.G. And L. MagaArd Geographic and seasonal distributions of Brunt-Vaisala frequency and Rossby radii in the North Pacific and North Atlantic. J. Phys. Oceanogr. 14 (1984), 294-317.

4 Flierl G.R., Malanote-Rizzoli P. and N.J. Zabusky: Nonlinear waves and coherent vortex structures in barotropic $\beta$-plane jets. J. Phys. Oceanogr. 17 (1987), 1408-1438.

5 Flierl G.R., Carton X.J. and C. Messager Vortex formation by unstable oceanic jets. Proceedings of the 3rd IWVF, this volume (1998)

6 Kontoyannis H. And D.R. Watts: Observations on the variability of the Gulf-Stream path between $74^{\circ} \mathrm{W}$ and $70^{\circ} \mathrm{W}$. J. Phys. Oceanogr. 24 (1994), 1999-2013.

7 LEE S. AND Held I.M. Subcritical instability and hysteresis in a two-layer model. J. Atm. Sci. 48 (1990), 1071-1077.

8 LEE T.: Variability of the Gulf-Stream path as observed from satellite infrared images. PhD dissertation, University of Rhode Island (1994), 188 pp.

9 Watts, R.: "Gulf-Stream variability". In "Eddies in Marine Sciences" (Ed. A.R. Robinson), SpringerVerlag (1983), 114-143. 


\section{Tables}

\begin{tabular}{|c|c|c|c|}
\hline Ocean jet & Azores current & Gulf-Stream & North Atlantic current \\
\hline$f * 10^{5}\left(s^{-1}\right)$ & 8.15 & 9.4 & 11.2 \\
\hline$H_{1}(m)$ & 700 & 800 & 800 \\
\hline$H(m)$ & 4200 & 4000 & 4000 \\
\hline$g^{\prime}\left(m \cdot s^{-2}\right.$ & 0.012 & 0.012 & 0.0035 \\
\hline$R_{d}(k m)$ & 32 & 30 & 15 \\
\hline$L(k m)$ & 50 & 30 & 40 \\
\hline$U_{1}\left(m \cdot s^{-1}\right)$ & 0.3 & 1.0 & 0.5 \\
\hline$R o$ & 0.07 & 0.3 & 0.11 \\
\hline$F r$ & 0.11 & 0.36 & 0.33 \\
\hline$B u$ & 0.4 & 0.7 & 0.1 \\
\hline$R o / B u$ & 0.17 & 0.42 & 1.1 \\
\hline
\end{tabular}

Table 1: Physical and geometrical characteristics of the North Atlantic ocean jets studied in the paper (Emery et al., 1984; Watts, 1983). 